\title{
Article
}

\section{On the Approximation by Balázs-Szabados Operators}

\author{
Adrian Holhoş \\ Department of Mathematics, Technical University of Cluj-Napoca, Str. Memorandumului 28, \\ RO-400114 Cluj-Napoca, Romania; Adrian.Holhos@math.utcluj.ro
}

check for updates

Citation: Holhoş, A. On the Approximation by Balázs-Szabados Operators. Mathematics 2021, 9, 1588. https://doi.org/10.3390/math9141588

Academic Editor: Clemente Cesarano

Received: 15 June 2021

Accepted: 5 July 2021

Published: 6 July 2021

Publisher's Note: MDPI stays neutral with regard to jurisdictional claims in published maps and institutional affiliations.

\begin{abstract}
We present three new approximation properties of the Balázs-Szabados operators. Firstly, we prove that, in certain cases, these operators approximate some super-exponential functions on compact intervals. Next, we provide a new estimate of the error of approximation using a suitable modulus of continuity. Finally, we characterize the functions which can be uniformly approximated in the weighted norm of polynomial weight spaces.
\end{abstract}

Keywords: positive linear operators; Balázs operators; rational Bernstein type operators; weighted spaces; modulus of continuity; tail inequality; super-exponential functions

MSC: 41A36; 41A25; 41A20

\section{Introduction}

The study of positive linear operators is an important research area in approximation theory. The problem of Weierstrass [1,2] to approximate continuous functions using a sequence of polynomials initiated this research field, but the systematic study of these operators began only in the 1960s with the books of Korovkin [3,4] and Lorentz [5]. Korovkin has discovered simple conditions to verify if a sequence of positive linear operators forms an approximation process, and since then, there have been constructed many operators. One such example is the sequence of operators defined for $\beta \in(0,1)$ by

$$
R_{n}^{[\beta]}(f, x)=\frac{1}{\left(1+n^{\beta-1} x\right)^{n}} \sum_{k=0}^{n}\left(\begin{array}{l}
n \\
k
\end{array}\right)\left(n^{\beta-1} x\right)^{k} \cdot f\left(\frac{k}{n^{\beta}}\right), \text { for } n \geq 1, x \in[0, \infty) .
$$

They were introduced in 1975 by K. Balázs [6]. She proved some pointwise approximation results for the particular value $\beta=2 / 3$. In 1982, Balázs and Szabados [7] extended the study for $\beta \in(0,2 / 3]$ and investigated both the pointwise and the uniform approximation. In 1984, Totik [8] considered $\beta \in(0,1)$ and provided the saturation properties of these operators. Many more articles [9-43] have appeared since then, presenting different new properties and generalizing the form of the operators. The vast majority refer to the operators defined by (1) as the Balázs-Szabados operators.

In this paper, we first present a new approximation result on compact intervals, extending the space of functions to be approximated. Balázs [6] showed that operators (1) approximate, on compact intervals, continuous functions with exponential growth. We prove that for certain values of $\beta$, operators (1) approximate even functions with superexponential growth. This fact is surprising for two reasons. Many classical operators approximate only functions with an exponential growth or some other fixed growth of exponential type. In our case, the growth can be enlarged indefinitely, in some sense, and this is correlated with the proper choice of the parameter $\beta$. Another interesting thing about the Balázs-Szabados operators is that they are built using only a finite sum and not a series and, so, the possibility of approximating functions with such a high growth comes as a surprise. This result is presented in Theorem 1 of Section 2 . The key ingredient of its proof is Lemma 1, which contains an inequality similar to the tail inequalities for the probability 
distributions. We also present, in Remark 3, an example of a super-exponential function which cannot be approximated by the operators (1) and, thus, we correct the recent result of ([39], Theorem 1), which gave the impression that every continuous function defined on $[0, \infty)$ can be approximated on compacts by the operators $R_{n}^{[\beta]}$, although the author mentions at the beginning of the article, when he defines the operators, that "continuous functions defined on $\mathbb{R}_{+}$satisfying a certain growth condition" are considered.

In Section 3, we present a new estimate of the rate of approximation by using a suitable modulus of continuity. In the literature, there are some estimations of this rate, but they are valid only for some values of $\beta$. We have obtained an estimation that is valid for every choice of $\beta \in(0,1)$. In addition, this estimation of the rate is uniform, uses only one modulus of continuity, and the constant in front of the modulus is explicit. We have used a modulus of continuity which is appropriate for continuous functions with a finite limit at infinity because it is known that these are precisely the functions that can be uniformly approximated by $R_{n}^{[\beta]}$ (see $[7,8]$ ). We must remark that better estimations can be obtained for specific values of the parameter $\beta$ and the value $\beta=1 / 2$ gives the best rate (see also the paper of Totik [8] which has arrived at the same conclusion).

In Section 4, we give a characterization of the functions which can be uniformly approximated in polynomial weight spaces. It is known that polynomial functions of degree $m$ are mapped by $R_{n}^{\beta}$ into rational functions with a growth not larger than a polynomial function of degree $m$ (see [16]), but I could not find a result in the literature that specifies the functions that can be uniformly approximated in the polynomial weight space. Recently, by considering a generalization of the operators (1), Agratini ([39], Theorem 5) has given a negative result by presenting an example of a function which cannot be approximated in the weighted norm. He has estimated the error of approximation, too, but only pointwise. Our results from Lemma 3 and Theorem 3 complete Agratini's results, characterizing the functions that can be uniformly approximated in the weighted norm.

We present now some notations. Let $I=[0, \infty)$. A function $w: I \rightarrow(0, \infty)$ will be called weight. The space of all functions $f: I \rightarrow \mathbb{R}$ with the property that there is $M>0$ such that

$$
|f(x)| \leq M \cdot w(x) \text {, for every } x \in I
$$

is called weight space or weighted space and is denoted by $B_{w}(I)$. Some authors prefer the "big O" notation to express the growth rate of a function. In this case, $f \in B_{w}(I)$ is equivalent to $f(x)=\mathcal{O}(w(x))$. The space $B_{w}(I)$ is a normed space, endowed with the $w$-norm

$$
\|f\|_{w}=\sup _{x \in I} \frac{|f(x)|}{w(x)} .
$$

We will denote by $C_{w}(I)$ the space of functions from $B_{w}(I)$ which are continuous on $I$.

\section{Approximation on Compact Intervals of Super-Exponential Functions}

The following lemma is very important in proving approximation results for the operators of Balázs and Szabados for functions with a high growth. For $y_{n}=1$, this is in fact a tail inequality for the probability distribution attached to the operators (1). We improve the idea used by Cernoff [44] to prove such inequalities (see also [45]).

Lemma 1. Let $\beta \in(0,1), x \in[0, \infty)$ and $\delta>0$ be given and consider $y_{n}>0$ such that $y_{n}\left(1-x n^{\beta-1}\right) \leq e^{\frac{1}{\delta n^{\beta}}}$, for all $n \geq n_{0}$, for some $n_{0} \in \mathbb{N}$. Then, for all $n \geq n_{0}$, we have

$$
\sum_{k \geq(x+\delta) n^{\beta}}^{n}\left(\begin{array}{l}
n \\
k
\end{array}\right) \frac{\left(x y_{n} n^{\beta-1}\right)^{k}}{\left(1+x n^{\beta-1}\right)^{n}} \leq C(x, \delta) \cdot \frac{y_{n}^{(x+\delta) n^{\beta}}}{n^{\beta}} \cdot e^{-x \delta \cdot n^{2 \beta-1}},
$$

where $C(x, \delta)=\frac{x e^{\frac{1-x-\delta+x e^{1 / \delta}}{\delta}}}{\delta^{2}}\left(\frac{x e^{\frac{1}{\delta}}}{\delta^{2}}+1\right)$. 
Proof. For $x=0$, the inequality (2) holds true. Let $x>0$ and denote $s_{n}=x n^{\beta-1}$. If $s_{n} \geq 1$, we have $k \geq n s_{n}+\delta n^{\beta}>n$, so the sum from (2) reduces to 0 and (2) is true. Consider now the case $s_{n}<1$. We have for every $t_{n} \geq 0$

$$
\begin{aligned}
\sum_{k \geq(x+\delta) n^{\beta}}^{n}\left(\begin{array}{l}
n \\
k
\end{array}\right) \frac{\left(y_{n} s_{n}\right)^{k}}{\left(1+s_{n}\right)^{n}} & \leq \sum_{k \geq(x+\delta) n^{\beta}}^{n}\left(\begin{array}{l}
n \\
k
\end{array}\right) \frac{\left(y_{n} s_{n}\right)^{k}}{\left(1+s_{n}\right)^{n}} \cdot e^{t_{n}\left(k-x n^{\beta}-\delta n^{\beta}\right)} \cdot\left(\frac{k-x n^{\beta}}{\delta n^{\beta}}\right)^{2} \\
& \leq \frac{e^{-t_{n} n s_{n}-t_{n} \delta n^{\beta}}}{\delta^{2}\left(1+s_{n}\right)^{n}} \sum_{k=0}^{n}\left(\begin{array}{l}
n \\
k
\end{array}\right)\left(y_{n} s_{n} e^{t_{n}}\right)^{k}\left(\frac{k}{n^{\beta}}-x\right)^{2}
\end{aligned}
$$

Using the same reasoning as in ([6], Lemma 2.1), we have for every $a, b>0$ :

$$
\begin{aligned}
\sum_{k=0}^{n}\left(\begin{array}{l}
n \\
k
\end{array}\right) a^{k}\left(\frac{k}{b}-x\right)^{2} & =\frac{1}{b^{2}} \sum_{k=0}^{n} k^{2}\left(\begin{array}{l}
n \\
k
\end{array}\right) a^{k}-\frac{2 x}{b} \sum_{k=0}^{n} k\left(\begin{array}{l}
n \\
k
\end{array}\right) a^{k}+x^{2} \sum_{k=0}^{n}\left(\begin{array}{l}
n \\
k
\end{array}\right) a^{k} \\
& =\frac{a^{2} n^{2}+a n}{b^{2}}(1+a)^{n-2}-\frac{2 x a n}{b}(1+a)^{n-1}+x^{2}(1+a)^{n} \\
& =(1+a)^{n}\left[\left(x-\frac{a n}{b(1+a)}\right)^{2}+\frac{a n}{b^{2}(1+a)^{2}}\right] .
\end{aligned}
$$

This implies that

$$
\begin{aligned}
& \sum_{k \geq(x+\delta) n^{\beta}}^{n}\left(\begin{array}{l}
n \\
k
\end{array}\right) \frac{\left(y_{n} s_{n}\right)^{k}}{\left(1+s_{n}\right)^{n}} \\
& \quad \leq \frac{e^{-t_{n}\left(n s_{n}+\delta n^{\beta}\right)}\left(1+y_{n} s_{n} e^{t_{n}}\right)^{n}}{\delta^{2}\left(1+s_{n}\right)^{n}}\left[x^{2}\left(\frac{y_{n} e^{t_{n}}}{1+y_{n} s_{n} e^{t_{n}}}-1\right)^{2}+\frac{n^{1-2 \beta} y_{n} s_{n} e^{t_{n}}}{\left(1+y_{n} s_{n} e^{t_{n}}\right)^{2}}\right],
\end{aligned}
$$

for all $t_{n} \geq 0$. We choose $t_{n}=\frac{1}{\delta n^{\beta}}-\ln \left[y_{n}\left(1-s_{n}\right)\right]$. Let us observe that for all $n \geq n_{0}$ we have $t_{n} \geq 0$ and

$$
\begin{aligned}
& e^{-t_{n}}=y_{n}\left(1-s_{n}\right) e^{-\frac{1}{\delta n^{\beta}}} \\
& e^{-t_{n}\left(n s_{n}+\delta n^{\beta}\right)}=y_{n}^{(x+\delta) n^{\beta}} \cdot\left(1-s_{n}\right)^{n s_{n}+\delta n^{\beta}} \cdot e^{-\frac{x+\delta}{\delta}} \\
& \left(1+y_{n} s_{n} e^{t_{n}}\right)^{n}=\frac{\left(1+s_{n} e^{\frac{1}{\delta n^{\beta}}}-s_{n}\right)^{n}}{\left(1-s_{n}\right)^{n}} \leq \frac{e^{n s_{n}\left(e^{\frac{1}{\delta n^{\beta}}}-1\right)}}{\left(1-s_{n}\right)^{n}} \leq \frac{e^{\frac{x e^{\frac{1}{\delta}} \delta}{\delta}}}{\left(1-s_{n}\right)^{n}} \\
& \frac{n^{1-2 \beta} y_{n} s_{n} e^{t_{n}}}{\left(1+y_{n} s_{n} e^{t_{n}}\right)^{2}}=\frac{n^{1-2 \beta} s_{n} e^{\frac{1}{\delta n^{\beta}}}\left(1-s_{n}\right)}{\left(1+s_{n}\left(e^{\frac{1}{\delta n^{\beta}}}-1\right)\right)^{2}} \leq n^{1-2 \beta} s_{n} e^{\frac{1}{\delta n^{\beta}}} \leq \frac{x e^{\frac{1}{\delta}}}{n^{\beta}} \\
& x^{2}\left(\frac{y_{n} e^{t_{n}}}{1+y_{n} s_{n} e^{t_{n}}}-1\right)^{2} \leq x^{2}\left(y_{n}\left(1-s_{n}\right) e^{t_{n}}-1\right)^{2}=x^{2}\left(e^{\frac{1}{\delta n^{\beta}}}-1\right)^{2} \leq \frac{x^{2} e^{\frac{2}{\delta}}}{\delta^{2} n^{2 \beta}} .
\end{aligned}
$$

We deduce that

$$
\sum_{k \geq(x+\delta) n^{\beta}}^{n}\left(\begin{array}{l}
n \\
k
\end{array}\right) \frac{\left(y_{n} s_{n}\right)^{k}}{\left(1+s_{n}\right)^{n}} \leq C(x, \delta) \cdot \frac{y_{n}^{(x+\delta) n^{\beta}}}{n^{\beta}} \cdot \frac{\left(1-s_{n}\right)^{n s_{n}+\delta n^{\beta}-n}}{\left(1+s_{n}\right)^{n}} .
$$

With the notation $c=\delta / x>0$, it remains to prove that

$$
\frac{\left(1-s_{n}\right)^{\delta n^{\beta}+n s_{n}-n}}{\left(1+s_{n}\right)^{n}}=e^{n\left(c s_{n}+s_{n}-1\right) \ln \left(1-s_{n}\right)-n \ln \left(1+s_{n}\right)}<e^{-n c s_{n}^{2}} .
$$


Consider the function $H(u)=(c u+u-1) \ln (1-u)-\ln (1+u)+c u^{2}$ and let us show that $H(u)<0$, for every $u \in(0,1)$. Indeed,

$$
\begin{aligned}
H^{\prime}(u) & =(c+1) \ln (1-u)+\frac{c u+u-1}{u-1}-\frac{1}{u+1}+2 c u, \\
H^{\prime \prime}(u) & =-\frac{c+1}{1-u}-\frac{c}{(1-u)^{2}}+\frac{1}{(u+1)^{2}}+2 c \\
H^{(3)}(u) & =-\frac{c+1}{(1-u)^{2}}-\frac{2 c}{(1-u)^{3}}-\frac{2}{(u+1)^{3}}<0 .
\end{aligned}
$$

Because $H^{\prime \prime}(0)=H^{\prime}(0)=H(0)=0$, we deduce $H(u)<0$, for every $u \in(0,1)$.

Lemma 2. Consider $w(x)=e^{\alpha x^{a}}$ for $\alpha \geq 0, a \geq 1$ and $1>\beta \geq \frac{a}{a+1}$. For every compact interval $K \subset[0, \infty)$, we have

$$
\lim _{n \rightarrow \infty} R_{n}^{[\beta]}(w, x)=w(x), \quad \text { uniformly on } K \text {. }
$$

Proof. Let $K=[m, M]$ be a compact interval included in $[0, \infty)$. Consider $\delta>0$ such that $\alpha \leq 1 / \delta$ and define

$$
p(x)=\left\{\begin{array}{ll}
w(x), & x \leq M+\delta \\
w(M+\delta), & x>M+\delta
\end{array} \quad \text { and } r(x)= \begin{cases}0, & x \leq M+\delta \\
w(x)-w(M+\delta), & x>M+\delta\end{cases}\right.
$$

The function $w$ can be decomposed into $w(x)=p(x)+r(x)$ and so

$$
\left|R_{n}^{[\beta]}(w, x)-w(x)\right| \leq\left|R_{n}^{[\beta]}(p, x)-p(x)\right|+\left|R_{n}^{[\beta]}(r, x)-r(x)\right| .
$$

We prove that both $p$ and $r$ can be uniformly approximated on K. Formula ([6], (2.2)) proves that $R_{n}^{[\beta]}(1, x)=1$, and using an idea of Shisha-Mond [46], the error of approximation of $p$ can be estimated using the modulus of continuity by

$$
\left|R_{n}^{[\beta]}(p, x)-p(x)\right| \leq 2 \cdot \omega\left(p, \sqrt{R_{n}^{[\beta]}\left(|t-x|^{2}, x\right)}\right) .
$$

Formula (2.4) from [6] gives

$$
R_{n}^{[\beta]}\left(|t-x|^{2}, x\right)=\frac{x^{4} n^{2 \beta-2}+x n^{-\beta}}{\left(1+x n^{\beta-1}\right)^{2}} \leq M^{4} n^{2 \beta-2}+M n^{-\beta},
$$

for every $x \in K$ and $\beta \in(0,1)$ and proves that $R_{n}^{[\beta]}\left(|t-x|^{2}, x\right)$ converges to 0 uniformly on $K$. Because $p$ has finite limit at infinity, it is uniformly continuous on $[0, \infty)$ and, so $\omega\left(p, \sqrt{R_{n}^{[\beta]}\left(|t-x|^{2}, x\right)}\right)$ tends uniformly to 0 on $K$. This proves the uniform convergence of $R_{n}^{[\beta]} p$ toward $p$ on $K$.

Now, for every $x \in K$

$$
\begin{aligned}
\left|R_{n}^{[\beta]}(r, x)-r(x)\right|=R_{n}^{[\beta]}(r, x) & \leq \sum_{\frac{k}{n^{\beta}}>M+\delta}^{n}\left(\begin{array}{l}
n \\
k
\end{array}\right) \frac{\left(n^{\beta-1} x\right)^{k}}{\left(1+n^{\beta-1} x\right)^{n}} \cdot e^{\alpha\left(\frac{k}{n^{\beta}}\right)^{a}} \\
& \leq \sum_{\frac{k}{n^{\beta}} \geq x+\delta}^{n}\left(\begin{array}{l}
n \\
k
\end{array}\right) \frac{\left(n^{\beta-1} x\right)^{k}}{\left(1+n^{\beta-1} x\right)^{n}} \cdot e^{\alpha k n^{a-1-a \beta}} .
\end{aligned}
$$


We apply Lemma 1 for $y_{n}=e^{\alpha n^{a-1-a \beta}}$. The inequality $y_{n}\left(1-x n^{\beta-1}\right) \leq e^{\frac{1}{\delta n \beta}}$ is equivalent to $n^{1-\beta}\left(1-e^{\frac{1}{\delta n^{\beta}}-\alpha n^{a-1-a \beta}}\right) \leq x$. However, this is true for sufficiently large $n$, since

$$
\begin{aligned}
\lim _{n \rightarrow \infty} n^{1-\beta}\left(1-e^{\frac{1}{\delta n^{\beta}}-\alpha n^{a-1-a \beta}}\right) & =\lim _{n \rightarrow \infty} n^{1-\beta} \cdot\left(\alpha n^{a-1-a \beta}-\frac{n^{-\beta}}{\delta}\right) \\
& =\lim _{n \rightarrow \infty}\left(\alpha \cdot n^{a-(a+1) \beta}-\frac{1}{\delta} \cdot n^{1-2 \beta}\right) \leq 0 .
\end{aligned}
$$

The error of approximation of the function $r$ is bounded by

$$
\left|R_{n}^{[\beta]}(r, x)-r(x)\right| \leq \frac{x}{\delta^{2} n^{\beta}} \cdot y_{n}^{(x+\delta) n^{\beta}} \cdot e^{-x \delta \cdot n^{2 \beta-1}} \leq \frac{C(M, \delta)}{n^{\beta}} \cdot e^{\alpha(M+\delta) n^{\beta+a-1-a \beta}-m \delta \cdot n^{2 \beta-1}} .
$$

Because $\beta \geq 1 / 2$ and $\beta+a-1-a \beta \leq 2 \beta-1$ we obtain that $R_{n}^{[\beta]} r$ converges uniformly to $r$ on $K$, and the proof of the lemma is complete.

Remark 1. For $a=1$ the result of Lemma 2 is true for every $\beta \in(0,1)$. Indeed, we have

$$
R_{n}^{[\beta]}\left(e^{\alpha t}, x\right)=\left(\frac{1+x n^{\beta-1} e^{\frac{\alpha}{n^{\beta}}}}{1+x n^{\beta-1}}\right)^{n} .
$$

For every $x \in[m, M]$, using $(1+u)^{n} \leq e^{n u}$ and $e^{u}-1 \leq u e^{u}$, we get

$$
\left(\frac{1+x n^{\beta-1} e^{\frac{\alpha}{n^{\beta}}}}{1+x n^{\beta-1}}\right)^{n} \leq\left(\frac{1+M n^{\beta-1} e^{\frac{\alpha}{n^{\beta}}}}{1+M n^{\beta-1}}\right)^{n} \leq e^{\frac{M n^{\beta}\left(e^{\left.\alpha n^{\beta}-1\right)}\right.}{1+M n^{\beta-1}}} \leq e^{\alpha M e^{\alpha}} .
$$

Now, using $|u-v| \leq|\ln u-\ln v| \cdot \max (u, v)$ we evaluate the error in approximating the exponential function

$$
\begin{aligned}
\left|R_{n}^{[\beta]}\left(e^{\alpha t}, x\right)-e^{\alpha x}\right| & \leq\left|n \ln \left(\frac{1+x n^{\beta-1} e^{\frac{\alpha}{n^{\beta}}}}{1+x n^{\beta-1}}\right)-\alpha x\right| \cdot \max \left(\left(\frac{1+x n^{\beta-1} e^{\frac{\alpha}{n^{\beta}}}}{1+x n^{\beta-1}}\right)^{n}, e^{\alpha x}\right) \\
& \leq\left|n \ln \left(\frac{1+x n^{\beta-1} e^{\frac{\alpha}{n^{\beta}}}}{1+x n^{\beta-1}}\right)-\alpha x\right| \cdot e^{\alpha M e^{\alpha}}
\end{aligned}
$$

For the logarithm we use $\frac{u}{1+u} \leq \ln (1+u) \leq u$ and we obtain

$$
\frac{x n^{\beta}\left(e^{\frac{\alpha}{n^{\beta}}}-1\right)}{1+x n^{\beta-1} e^{\frac{\alpha}{n^{\beta}}}} \leq n \ln \left(\frac{1+x n^{\beta-1} e^{\frac{\alpha}{n^{\beta}}}}{1+x n^{\beta-1}}\right) \leq \frac{x n^{\beta}\left(e^{\frac{\alpha}{n^{\beta}}}-1\right)}{1+x n^{\beta-1}} .
$$

Using the inequalities $u \leq e^{u}-1 \leq u e^{u}$ we have

$$
\frac{x n^{\beta}\left(e^{\frac{\alpha}{n^{\beta}}}-1\right)}{1+x n^{\beta-1}}-\alpha x \leq \frac{\alpha x e^{\frac{\alpha}{n^{\beta}}}}{1+x n^{\beta-1}}-\alpha x \leq \frac{\alpha x\left(e^{\frac{\alpha}{n^{\beta}}}-1\right)}{1+x n^{\beta-1}} \leq \frac{\alpha^{2} x}{n^{\beta}} \leq \frac{\alpha^{2} M}{n^{\beta}}
$$

and

$$
\frac{x n^{\beta}\left(e^{\frac{\alpha}{n^{\beta}}}-1\right)}{1+x n^{\beta-1} e^{\frac{\alpha}{n^{\beta}}}}-\alpha x \geq \frac{\alpha x}{1+x n^{\beta-1} e^{\frac{\alpha}{n^{\beta}}}}-\alpha x \geq-\frac{\alpha x^{2} n^{\beta-1} e^{\frac{\alpha}{n^{\beta}}}}{1+x n^{\beta-1} e^{\frac{\alpha}{n^{\beta}}}} \geq-\alpha M^{2} n^{\beta-1} e^{\alpha}
$$


Finally, for every $x \in[m, M]$ we obtain

$$
\left|R_{n}^{[\beta]}\left(e^{\alpha t}, x\right)-e^{\alpha x}\right| \leq \alpha M \max \left(\alpha n^{-\beta}, M n^{\beta-1} e^{\alpha}\right) \cdot e^{\alpha M e^{\alpha}} .
$$

Theorem 1. Consider $w(x)=e^{\alpha x^{a}}$ with $\alpha \geq 0, a \geq 1$ and $1>\beta \geq \frac{a}{a+1}$. For every compact interval $K \subset[0, \infty)$ and for every $f \in C_{w}(I)$ we have

$$
\lim _{n \rightarrow \infty} R_{n}^{[\beta]}(f, x)=f(x), \quad \text { uniformly on } K \text {. }
$$

Proof. Let $\varepsilon>0$. Consider the compact interval $J=[\min K-\varepsilon, \max K+\varepsilon] \cap[0, \infty)$. Because $f / w$ and $w$ are continuous on the compact $J$, there is $\eta>0$ such that for every $t, x \in J$ with $|t-x|<\eta$ we have $|(f / w)(t)-(f / w)(x)|<\varepsilon$ and $|w(t)-w(x)|<\varepsilon$. Let us define $\delta=\min (\eta, \varepsilon)$. For every $x \in K$ and $t \in I$ such that $|t-x|<\delta$, we have $t, x \in J$. So

$$
\left|\frac{f(t)}{w(t)}-\frac{f(x)}{w(x)}\right|<\varepsilon \quad \text { and } \quad|w(t)-w(x)|<\varepsilon .
$$

For every $x \in K$ and $t \in I$ such that $|t-x| \geq \delta$, we can write

$$
\left|\frac{f(t)}{w(t)}-\frac{f(x)}{w(x)}\right| \leq 2\|f\|_{w} \leq 2\|f\|_{w} \cdot \frac{|t-x|^{2}}{\delta^{2}}
$$

and

$$
|w(t)-w(x)| \leq w(t)+w(x) \leq w(t)-w(x)+2 w(x) \leq w(t)-w(x)+2 w(x) \cdot \frac{|t-x|^{2}}{\delta^{2}} .
$$

We have proved that, for every $t \in I$ and $x \in K$, we have

$$
\left|\frac{f(t)}{w(t)}-\frac{f(x)}{w(x)}\right|<\varepsilon+2\|f\|_{w} \cdot \frac{|t-x|^{2}}{\delta^{2}}
$$

and

$$
|w(t)-w(x)|<\varepsilon+w(t)-w(x)+2 w(x) \cdot \frac{|t-x|^{2}}{\delta^{2}} .
$$

Using the above inequalities and

$$
|f(t)-f(x)| \leq \frac{|f(t)|}{w(t)} \cdot|w(t)-w(x)|+w(x) \cdot\left|\frac{f(t)}{w(t)}-\frac{f(x)}{w(x)}\right|
$$

and applying the operators $R_{n}^{[\beta]}$ we deduce that

$$
\begin{aligned}
\left|R_{n}^{[\beta]}(f, x)-f(x)\right| \leq & \|f\|_{w}\left(\varepsilon+\left|R_{n}^{[\beta]}(w, x)-w(x)\right|+\frac{2 w(x)}{\delta^{2}} \cdot R_{n}^{[\beta]}\left(|t-x|^{2}, x\right)\right) \\
& +w(x) \cdot\left(\varepsilon+\frac{2\|f\|_{w}}{\delta^{2}} R_{n}^{[\beta]}\left(|t-x|^{2}, x\right)\right) .
\end{aligned}
$$

Using Lemma 2 and the inequality $w(x) \leq w(\max K)$ for every $x \in K$ and the fact that $R_{n}^{[\beta]}\left(|t-x|^{2}, x\right)$ converges uniformly on $K$, we obtain that $R_{n}^{[\beta]} f$ converges uniformly to $f$ on $K$.

Remark 2. The result of Theorem 1 is valid for $\beta \geq 1 / 2$. For a given $\beta \in[1 / 2,1)$, the growth of the functions cannot be greater than $w(x)=e^{\alpha x^{a}}$ with $\alpha \geq 0$ and $a \leq \frac{\beta}{1-\beta}<\frac{1}{1-\beta}$. 
For $a=1$ the result is valid for every $\beta \in(0,1)$. For $\beta=\frac{2}{3}$ and $a=1$ the result is known from [6]. In [7], the authors proved the approximation property of the operators (1) for $\beta \in(0,2 / 3$ ] and the subspace of uniformly continuous functions on I.

Remark 3. There are super-exponential functions which cannot be approximated. Take for example $g(x)=e^{x^{\frac{1}{1-\beta}+\varepsilon}}$, with an arbitrary $\varepsilon>0$. Considering only the last term of the sum which defines the operators $R_{n}^{[\beta]}$, we have

$$
R_{n}^{[\beta]}(g, x) \geq \frac{\left(n^{\beta-1} x\right)^{n}}{\left(1+n^{\beta-1} x\right)^{n}} \cdot g\left(n^{1-\beta}\right)=\left(n^{\beta-1} x\right)^{n} \cdot\left(1+n^{\beta-1} x\right)^{-n} \cdot e^{n \cdot n^{(1-\beta) \varepsilon}} .
$$

Using the inequality $1+n^{\beta-1} x \leq e^{x n^{\beta-1}}$ we deduce that

$$
R_{n}^{[\beta]}(g, x) \geq\left(n^{\beta-1} x\right)^{n} \cdot e^{-x n^{\beta}} \cdot e^{n \cdot n^{(1-\beta) \varepsilon}}=e^{n \ln x-(1-\beta) n \ln n-x n^{\beta}+n \cdot n^{(1-\beta) \varepsilon}} .
$$

Now, it is not difficult to see that $\lim _{n \rightarrow \infty} R_{n}^{[\beta]}(g, x)=+\infty$, for every $x>0$ and $\beta \in(0,1)$.

Theorem 1 from [39] asserts that the sequence $\left(R_{n}^{[\beta]} f\right)$ converges to $f$ on compact sets for every $f \in C[0, \infty)$. However, the example given above proves that an approximation result cannot be true for continuous functions with an arbitrary growth. In order to be valid, such a result must impose some limitation on the growth of the function $f$.

\section{Estimation of the Rate of Uniform Approximation}

It is known from [7], that $R_{n}^{[\beta]} f$ approximate uniformly the continuous function $f$ on $I=[0, \infty)$ if, and only if, $f$ has a finite limit at infinity. In the same paper, an estimate of the rate of convergence was given using the modulus of continuity and a modulus at infinity. We will provide an estimate of the rate of convergence using the following modulus of continuity

$$
\omega^{*}(f, \delta)=\sup _{\substack{x, t \in I \\\left|e^{-t}-e^{-x}\right| \leq \delta}}|f(t)-f(x)|,
$$

introduced and studied in [47] (a particular case of the modulus introduced in [48-50]). This modulus is suitable for the uniform approximation of functions by the Balázs-Szabados operators, since $\omega^{*}(f, \cdot)$ tends to zero when its argument tends to zero and the function $f$ has a finite limit at infinity.

Theorem 2. Consider $\beta \in(0,1)$ and $f$ a bounded and continuous function defined on I having a finite limit at infinity. Then,

$$
\left\|R_{n}^{[\beta]} f-f\right\| \leq(1+e) \cdot \omega^{*}\left(f, \sqrt{\max \left(n^{\beta-1}, n^{-\beta}\right)}\right), \quad \text { for every } n \in \mathbb{N} .
$$

Proof. Using the properties of the modulus $\omega^{*}$ (see the proof of ([47], Theorem 2.1)), we obtain

$$
\left|R_{n}^{[\beta]}(f, x)-f(x)\right| \leq\left(1+\frac{1}{\delta_{n}^{2}} \cdot R_{n}^{[\beta]}\left(\left|e^{-t}-e^{-x}\right|^{2}, x\right)\right) \cdot \omega^{*}\left(f, \delta_{n}\right) .
$$

It remains to estimate in the uniform norm the following expression:

$$
R_{n}^{[\beta]}\left(\left|e^{-t}-e^{-x}\right|^{2}, x\right)=R_{n}^{[\beta]}\left(e^{-2 t}, x\right)-e^{2 x}-2 e^{-x}\left[R_{n}^{[\beta]}\left(e^{-t}, x\right)-e^{x}\right] .
$$


Because $e^{-x}$ is a convex function, applying Jensen inequality, we obtain

$$
R_{n}^{[\beta]}\left(e^{-t}, x\right) \geq e^{-R_{n}^{[\beta]}\left(e_{1}, x\right)}=e^{\frac{-x}{1+x n^{\beta-1}}} \geq e^{-x} .
$$

As a consequence, we have

$$
R_{n}^{[\beta]}\left(\left|e^{-t}-e^{-x}\right|^{2}, x\right) \leq R_{n}^{[\beta]}\left(e^{-2 t}, x\right)-e^{-2 x} .
$$

Let us denote

$$
\Delta_{n}(x)=R_{n}^{[\beta]}\left(e^{-2 t}, x\right)-e^{-2 x}=\left(\frac{1+x n^{\beta-1} e^{-\frac{2}{n^{\beta}}}}{1+x n^{\beta-1}}\right)^{n}-e^{-2 x}, \quad x \geq 0 .
$$

Because the limit

$$
\lim _{x \rightarrow \infty} \Delta_{n}(x)=e^{-2 n^{1-\beta}}
$$

is finite and $\Delta_{n}(x) \geq 0$, there is a sequence $\left(x_{n}\right)$ of positive numbers such that

$$
\max _{x \geq 0} \Delta_{n}(x)=\Delta_{n}\left(x_{n}\right) .
$$

This implies that $\Delta_{n}^{\prime}\left(x_{n}\right)=0$, which is equivalent to

$$
\left(\frac{1+x_{n} n^{\beta-1} e^{-\frac{2}{n^{\beta}}}}{1+x_{n} n^{\beta-1}}\right)^{n}=\frac{2 e^{-2 x_{n}}\left(1+x_{n} n^{\beta-1} e^{-\frac{2}{n^{\beta}}}\right)\left(1+x_{n} n^{\beta-1}\right)}{n^{\beta}\left(1-e^{-\frac{2}{n^{\beta}}}\right)} .
$$

It follows that

$$
\Delta_{n}(x) \leq \frac{2\left(1+x_{n} n^{\beta-1} e^{-\frac{2}{n^{\beta}}}\right)\left(1+x_{n} n^{\beta-1}\right)-n^{\beta}\left(1-e^{-\frac{2}{n^{\beta}}}\right)}{e^{2 x_{n}} n^{\beta}\left(1-e^{-\frac{2}{n^{\beta}}}\right)} .
$$

Using $1-e^{-u}>u-\frac{u^{2}}{2}$ for $u=2 n^{-\beta}>0$, we obtain

$$
\Delta_{n}(x) \leq \frac{2 x_{n} n^{\beta-1}\left(e^{-\frac{2}{n^{\beta}}}+1\right)+2 x_{n}^{2} n^{2 \beta-2} e^{-\frac{2}{n^{\beta}}}+2 n^{-\beta}}{e^{2 x_{n}} n^{\beta}\left(1-e^{-\frac{2}{n^{\beta}}}\right)} .
$$

Finally, using the inequality $\frac{u}{1-e^{-u}}<e$, for $u=2 n^{-\beta} \leq 2$ and $\frac{(1+u)^{2}}{e^{2 u}} \leq 1$, for $u=x_{n}>0$, we have

$$
\Delta_{n}(x) \leq e \max \left(n^{\beta-1}, n^{-\beta}\right) \cdot\left(\frac{2 x_{n}+x_{n}^{2}+1}{e^{2 x_{n}}}\right)<e \max \left(n^{\beta-1}, n^{-\beta}\right) .
$$

We choose $\delta_{n}=\sqrt{\max \left(n^{\beta-1}, n^{-\beta}\right)}$ in the first inequality of the proof.

Remark 4. The maximum rate of approximation is obtained for $\beta=\frac{1}{2}$. 


\section{Weighted Approximation in Polynomial Weight Spaces}

It is known from [16] that operators $R_{n}^{[\beta]}$ map a polynomial function of degree $m$ into a function with a growth not larger than a polynomial function of degree $m$. In the following lemma, we extend this result.

Lemma 3. For every $\beta \in(0,1), \alpha \geq 0, n \in \mathbb{N}$ and every $x \geq 0$ we have

$$
R_{n}^{[\beta]}\left(1+t^{\alpha}, x\right) \leq C_{\alpha}\left(1+x^{\alpha}\right),
$$

for some constant $C_{\alpha}>0$ independent of $n, \beta$ and $x$.

Proof. For $\alpha=0$, we have equality with $C_{0}=1$. For $\alpha>0$, let $m=\lceil\alpha\rceil \geq 1$. In ([16], Lemma 2) it was proved that

$$
R_{n}^{[\beta]}\left(t^{m}, x\right) \leq C_{m}\left(1+x^{m}\right), \quad \text { for every } x \geq 0,
$$

where $C_{m}=m \cdot \max _{1 \leq j \leq m} S(m, j)$ and $S(m, j)$ are the Stirling numbers of the second kind (see ([51], 24.1.4)).

Applying Hölder inequality we get

$$
R_{n}^{[\beta]}\left(1+t^{\alpha}, x\right)=1+R_{n}^{[\beta]}\left(t^{\alpha}, x\right) \leq 1+\left(R_{n}^{[\beta]}\left(t^{m}, x\right)\right)^{\frac{\alpha}{m}} \leq 1+\left(C_{m}\right)^{\frac{\alpha}{m}} \cdot\left(1+x^{m}\right)^{\frac{\alpha}{m}} .
$$

However, it is known that $u^{a}-v^{a} \leq(u-v)^{a}$, for $u \geq v$ and $a \in(0,1]$ (see for example ([52], Example 1.1.3)). We deduce that

$$
\left(1+x^{m}\right)^{\frac{\alpha}{m}}-\left(x^{m}\right)^{\frac{\alpha}{m}} \leq 1
$$

and

$$
R_{n}^{[\beta]}\left(1+t^{\alpha}, x\right) \leq 1+\left(C_{m}\right)^{\frac{\alpha}{m}}\left(1+x^{\alpha}\right) \leq\left[1+\left(C_{m}\right)^{\frac{\alpha}{m}}\right]\left(1+x^{\alpha}\right) .
$$

Lemma 3 proves that $R_{n}^{\beta} w$ belongs to the space $C_{w}(I)$, for $w(x)=1+x^{\alpha}$ with $\alpha \geq 0$. We give now a complete characterization of the functions which can be approximated in the $w$-norm.

Theorem 3. Let $\beta \in(0,1)$. Consider $\alpha>0$ and $w(x)=1+x^{\alpha}, x \in$ I. If $f \in C_{w}(I)$, then

$$
\lim _{n \rightarrow \infty}\left\|R_{n}^{[\beta]} f-f\right\|_{w}=0
$$

if and only if

$$
\lim _{x \rightarrow \infty} \frac{f(x)}{1+x^{\alpha}}=0
$$

Proof. Using the definition of the operators, we deduce that

$$
\lim _{x \rightarrow \infty} \frac{R_{n}^{[\beta]}(f, x)}{(1+x)^{\alpha}}=0 .
$$

For the "if" part, we suppose that

$$
\lim _{x \rightarrow \infty} \frac{f(x)}{(1+x)^{\alpha}}=0 .
$$


We obtain

$$
\lim _{x \rightarrow \infty} \frac{\left|R_{n}^{[\beta]}(f, x)-f(x)\right|}{(1+x)^{\alpha}}=\lim _{x \rightarrow \infty} \frac{|f(x)|}{(1+x)^{\alpha}}=0 .
$$

Hence, for $\varepsilon>0$ there is $\delta>0$ such that for every $x>\delta$ and every $n \in \mathbb{N}$

$$
(1+x)^{-\alpha}\left|R_{n}^{[\beta]}(f, x)-f(x)\right|<\varepsilon
$$

For the compact interval $K=[0, \delta]$, we apply ([16], Theorem 2 ) or our Theorem 1 and deduce the existence of $n_{0} \in \mathbb{N}$ such that

$$
\left|R_{n}^{[\beta]}(f, x)-f(x)\right|<\varepsilon,
$$

for every $n \geq n_{0}$ and every $x \in K$. This proves that

$$
\sup _{x \geq 0}(1+x)^{-\alpha}\left|R_{n}^{[\beta]}(f, x)-f(x)\right|<\varepsilon .
$$

For the "only if" part, let us observe that

$$
\left\|R_{n}^{[\beta]} f-f\right\|_{w}=\sup _{x \geq 0} \frac{\left|R_{n}^{[\beta]}(f, x)-f(x)\right|}{(1+x)^{\alpha}} \geq \lim _{x \rightarrow \infty} \frac{\left|R_{n}^{[\beta]}(f, x)-f(x)\right|}{(1+x)^{\alpha}}=\lim _{x \rightarrow \infty} \frac{|f(x)|}{(1+x)^{\alpha}} .
$$

Applying the limit when $n$ tends to infinity, we obtain that $\lim _{x \rightarrow \infty} \frac{|f(x)|}{(1+x)^{\alpha}}=0$.

Remark 5. As Agratini [39] has remarked, we cannot approximate uniformly in the w-norm all the functions from the space $C_{w}(I)$, where $w(x)=1+x^{\alpha}$ and $I=[0, \infty), \alpha>0$. For $\alpha=2$, Agratini gave as an example the function $f(x)=x^{2}$, which cannot be uniformly approximated in the weighted w-norm by $R_{n}^{[\beta]} f$. Our result says that only those functions for which we have

$$
\lim _{x \rightarrow \infty} \frac{f(x)}{w(x)}=0
$$

can be uniformly approximated.

Funding: This research received no external funding.

Institutional Review Board Statement: Not applicable.

Informed Consent Statement: Not applicable.

Data Availability Statement: Not applicable.

Conflicts of Interest: The author declares no conflict of interest.

\section{References}

1. Weierstrass, K. Über die analytische Darstellbarkeit sogenannter willkürlicher Functionen einer reellen Veränderlichen. Sitzungsber. Akad. Berlin 1885, 633-639, 789-805. (In German)

2. Weierstrass, K. Sur la possibilité d'une représentation analytique des fonctions dites arbitraires d'une variable réelle. J. Math. Pures Appl. 1886, tome 2, 105-138. (In French)

3. Korovkin, P.P. Linear Operators and Approximation Theory; State Publishing, Physical and Mathematical Literature: Moscow, Russia, 1959. (In Russian)

4. Korovkin, P.P. Linear Operators and Approximation Theory; Russian Monographs and Texts on Advanced Mathematics and Physics; Hindustan Publishing Corporation: Delhi, India, 1960; Volume III.

5. Lorentz, G.G. Approximation of Functions; Holt, Rinhart and Winston, Inc.: New York, NY, USA, 1966.

6. Balázs, K. Approximation by Bernstein type rational functions. Acta Math. Hungar. 1975, 26, 123-134. [CrossRef]

7. Balázs, C.; Szabados, J. Approximation by Bernstein type rational functions II. Acta Math. Hungar. 1982, 40, 331-337. [CrossRef]

8. Totik, V. Saturation for Bernstein type rational functions. Acta Math. Hungar. 1984, 43, 219-250. [CrossRef] 
9. Balázs, K. Approximation by Bernstein type rational functions on the real axis. Acta Math. Hungar. 1985, 46, 195-204. [CrossRef]

10. della Vecchia, B. On some preservation and asymptotic relations of a rational operator. Facta Univ. Ser. Math. Inform. 1989, 4, 57-62.

11. Ispir, N. Approximation by modified Bernstein-Balazs type rational functions. Commun. Fac. Sci. Univ. Ank. Ser. A1 2000, 49, 87-93. [CrossRef]

12. Abel, U.; della Vecchia, B. Asymptotic approximation by the operators of K. Balázs and Szabados. Acta Sci. Math. 2000, 66, 137-145.

13. Agratini, O. An approximation process of Kantorovich type. Miskolc Math. Notes 2001, 2, 3-10. [CrossRef]

14. Atakut, C. On approximation by Balazs-Stancu type rational functions. Commun. Fac. Sci. Univ. Ank. Ser. A1 2001, 50, 95-105.

15. Ispir, N.; Atakut, C. Approximation by Balazs type rational functions in weighted spaces. Hadronic J. 2001,24 , $301-315$.

16. Agratini, O. On approximation properties of Balázs-Szabados operators and their Kantorovich extension. Korean J. Comput. Appl. Math. 2002, 9, 361-372. [CrossRef]

17. Dogru, O. On Bleimann, Butzer and Hahn type generalization of Balázs operators. Stud. Univ. Babeş-Bolyai Math. 2002, 47, 37-45.

18. Ispir, N.; Atakut, C. Approximation by generalized Balazs type rational functions. Int. J. Comput. Numer. Anal. Appl. 2003, 4, 297-316.

19. Atakut, C.; Ispir, N. On Bernstein type rational functions of two variables. Math. Slovaca 2004, 54, 291-301. [CrossRef]

20. Gupta, V.; Lupaş, A. On the rate of approximation for the Bézier variant of Kantorovich-Balazs operators. Gen. Math. 2004, $12,3-18$. [CrossRef]

21. Gupta, V.; Ispir, N. On the Bézier variant of generalized Kantorovich type Balazs operators. Appl. Math. Lett. 2005, 18, 1053-1061. [CrossRef]

22. Dogru, O. On statistical approximation properties of Stancu type bivariate generalization of $q$-Balázs-Szabados operators. In Proceedings of the International Conference on Numerical Analysis and Approximation Theory NAAT 2006, Cluj-Napoca, Romania, 5-8 July 2006; Casa Cărţii de Ştiinţă: Cluj-Napoca, Romania, 2006; pp. 179-194.

23. Gupta, V.; Ispir, N. On the Kantorovich variant of generalized Bernstein type rational functions. Demonstr. Math. 2006, 39, 117-130. [CrossRef]

24. Gupta, V.; Zeng, X.M. Rate of approximation for the Bézier variant of Balazs-Kantorovich operators. Math. Slovaca 2007, 57, 349-358. [CrossRef]

25. Gupta, V. Rate of approximation for the Balazs-Kantorovich-Bézier operators. Appl. Math. Comput. 2008, 199, 823-827. [CrossRef]

26. Gal, S. Approximation by Complex Bernstein and Convolution Type Operators; Series on Concrete and Applicable Mathematics: Volume 8; World Scientific Publishing Co Pte Ltd.: Singapore, 2009; pp. 139-148.

27. Atakut, C. On derivatives of Bernstein type rational functions of two variables. Appl. Math. Comput. 2011, 218, 673-677. [CrossRef]

28. Ispir, N.; Özkan, E.Y. Approximation properties of complex q-Balázs-Szabados operators in compact disks. J. Inequal. Appl. 2013, 2013, 361. [CrossRef]

29. Özkan, E.Y. Approximation properties of bivariate complex $q$-Balázs-Szabados operators of tensor product kind. J. Inequal. Appl. 2014, 2014, 20. [CrossRef]

30. Özkan, E.Y. Statistical Approximation Properties of $q$-Balázs-Szabados-Stancu Operators. Filomat 2014, 28, 1943-1952. [CrossRef]

31. Atakut, C.; Büyükyazici, I.; Serenbay, S.K. Approximation properties of Baskakov-Balazs type operators for functions of two variables. Miskolc Math. Notes 2015, 16, 667-678.

32. Deo, N.; Bhardwaj, N. A Better Error Estimation on Balázs Operators. Lobachevskii J. Math. 2015, 36, 9-14. [CrossRef]

33. Abel, U.; Agratini, O. On the variation detracting property of operators of Balázs and Szabados. Acta Math. Hungar. 2016, 150, 383-395. [CrossRef]

34. Mahmudov, N.I. Approximation Properties of the $q$-Balázs-Szabados Complex Operators in the Case $q \geq 1$. Comput. Methods Funct. Theory 2016, 16, 567-583. [CrossRef]

35. Özkan, E.Y. Approximation by Complex Bivariate Balázs-Szabados Operators. Bull. Malays. Math. Sci. Soc. 2016, 39, 1-16. [CrossRef]

36. Özkan, E.Y.; Ispir, N. Approximation by $(p, q)$-Analogue of Bálazs-Szabados Operators. Filomat 2018, 32, 2257-2271. [CrossRef]

37. Özkan, E.Y. Weighted Statistical Approximation of Kantorovich type $q$-Balazs-Szabados operators including the Riemann type q-integral. In Academic Researches in Mathematic and Sciences; Gece Kitapliği: Ankara, Turkey, 2018; pp. 9-19.

38. Özkan, E.Y. Approximation Properties of Kantorovich type q-Balázs-Szabados Operators. Demonstr. Math. 2019, 52, 10-19. [CrossRef]

39. Agratini, O. On a Class of Bernstein-Type Rational Functions. Numer. Funct. Anal. Optim. 2019, 41, 483-494. [CrossRef]

40. Hamal, H.; Sabancigil, P. Some approximation properties of new Kantorovich type $q$-analogue of Balázs-Szabados operators. J. Inequal. Appl. 2020, 2020, 159. [CrossRef]

41. Holhos, A. The product of two functions using positive linear operators. Constr. Math. Anal. 2020, 3, 64-74. [CrossRef]

42. Özkan, E.Y. Quantitative Estimates for the Tensor Product $(p, q)$-Balázs-Szabados Operators and Associated Generalized Boolean Sum Operators. Filomat 2020, 34, 779-793. [CrossRef]

43. Özkan, E.Y. Approximation by Fuzzy Bernstein Type Rational Functions. In Academic Studies in Science and Mathematics Sciences; Ucuncu, S.I., Soldatović, T.V., Eds.; Livre de Lyon: Lyon, France, 2020; pp. 71-86.

44. Chernoff, H. A measure of asymptotic efficiency for tests of a hypothesis based on the sum of observations. Ann. Math. Stat. 1952, 23, 493-507. [CrossRef] 
45. Boucheron, S.; Lugosi, G; Massart, P. Concentration Inequalities. A Nonasymptotic Theory of Independence; Oxford University Press: Oxford, UK, 2013.

46. Sisha, O.; Mond, B. The degree of convergence of sequences of linear positive operators. Proc. Natl. Acad. Sci. USA 1968, 60, 1196-1200. [CrossRef]

47. Holhoş, A. The rate of approximation of functions in an infinite interval by positive linear operators. Stud. Univ. Babeş-Bolyai Math 2010, 55, 133-142.

48. Holhoş, A. The rate of convergence of positive linear operators in weighted spaces. Automat. Comput. Appl. Math. 2008, 17, 239-246.

49. Holhoş, A. Uniform approximation by positive linear operators on noncompact intervals. Automat. Comput. Appl. Math. 2009, 18, 121-132.

50. Holhoş, A. Quantitative Estimates of Voronovskaya Type in Weighted Spaces. Results Math. 2018, 73, 53. [CrossRef]

51. Abramowitz, M.; Stegun, I.A. Handbook of Mathematical Functions with Formulas, Graphs, and Mathematical Tables; National Bureau of Standards, Applied Mathematics Series 55; Issued 1964, Tenth Printing; U.S. Government Printing Office: Washington, DC, USA, 1972.

52. Fiorenza, R. Hölder and Locally Hölder Continuous Functions, and Open Sets of Class $C^{k}, C^{k, \lambda}$; Birkhäuser: Cham, Switzerland, 2016. 